\section{GENRE}

en séries
Genre en séries

Cinéma, télévision, médias

5 | 2017

Masculinités imag(in)les 2

\title{
Des féminismes et des hommes ? Réflexions exploratoires sur la lente responsabilisation des personnages masculins de séries étatsuniennes
}

\section{Céline Morin}

\section{(2) OpenEdition}

Journals

Édition électronique

URL : http://journals.openedition.org/ges/826

DOI : $10.4000 /$ ges. 826

ISSN : 2431-6563

Éditeur

Presses universitaires de Bordeaux

Référence électronique

Céline Morin, « Des féminismes et des hommes ? Réflexions exploratoires sur la lente responsabilisation des personnages masculins de séries étatsuniennes », Genre en séries [En ligne], 5 | 2017, mis en ligne le 01 juin 2017, consulté le 22 mars 2021. URL : http://journals.openedition.org/ ges/826 ; DOI : https://doi.org/10.4000/ges.826

Ce document a été généré automatiquement le 22 mars 2021.

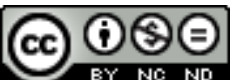

La revue Genre en séries est mise à disposition selon les termes de la Licence Creative Commons Attribution - Pas d'Utilisation Commerciale - Pas de Modification 4.0 International. 


\title{
Des féminismes et des hommes? Réflexions exploratoires sur la lente responsabilisation des personnages masculins de séries étatsuniennes
}

\author{
Céline Morin
}

1 Si les inégalités de genre ont très tôt été mises en scène dans les séries télévisées étatsuniennes, elles sont longtemps restées une affaire de femmes : nombreuses sont les stratégies qui, quarante ans durant, ont soigneusement évincé les personnages masculins des causes et des enjeux féministes. La division fonctionnaliste et le féminisme libéral ont tour à tour ramené la lutte antisexiste au genre féminin, bien aidés par une séparation franche entre sphère publique et sphère privée.

2 L'étude des formes amoureuses, dans leurs structures, leurs imaginaires et leurs codes communicationnels, est un terrain de premier plan pour comprendre la progressive responsabilisation féministe des personnages masculins: pour Beck et BeckGernsheim (1995), la modernité avancée redéploie des conflits sociaux dans la sphère privée, émancipant l'amour de la famille et de l'économie pour en faire le plus grand espoir contemporain d'épanouissement personnel. Cette détraditionnalisation explique la précarité grandissante des liens amoureux, ce qui ouvre à la fois de grandes angoisses mais aussi des possibilités jusqu'ici étouffées : exploration des désirs et des plaisirs sexuels, échanges de sphères d'activité, découvertes de nouveaux codes communicationnels, ou encore, bien sûr, performances de genre plus éclatées (Giddens, 1992). Les représentations de l'amour dans les séries télévisées, qui en offrent des définitions convergentes ou contradictoires, constituent ainsi l'un des terrains, sinon le terrain le plus propice à l'analyse des ondes de choc de l'émancipation féminine.

3 Ceci explique notre choix de n'évoquer que les séries télévisées étatsuniennes dont le personnage principal est une femme: ce choix a priori contradictoire a l'avantage crucial d'examiner les réponses qu'apportent les personnages masculins lorsqu'ils sont placés au cœur de l'émancipation féminine, des décentrements égalitaires qui, en toute 
logique, ont été incarnés par des héroïnes. Nous nous focaliserons sur les représentations de couples hétérosexuels, lesquels apparaissent comme les plus à même de résister à la représentation d'une compréhension inter-genre.

Cet article commence par un court retour historique sur près d'un demi-siècle de masculinités patriarcales, un retour essentiel pour comprendre les redéploiements des vingt dernières années qui constituent le noyau empirique de cette réflexion en ce qu'elles marquent la lente responsabilisation des personnages masculins : ceux-ci ne sont plus simplement désignés comme responsables des inégalités de genre, mais sont désormais inclus comme acteurs de l'égalité de genre. À compter des années 1990 en effet, les séries télévisées problématisent les asymétries que provoque le modèle romantique entre hommes et femmes et s'emparent des conséquences des émancipations féminines sur les performances de genre, les codes communicationnels et les attentes amoureuses et sexuelles des personnages masculins. La question fondamentale qui sous-tend ces représentations est le lien entre hommes et féminismes : longtemps tenus à l'écart de l'égalité de genre, sous prétexte que celle-ci serait le problème des femmes, les personnages masculins deviennent, parfois malgré eux, acteurs de ces transformations sociales à compter des années 2000. On voit dans les récentes séries étatsuniennes affleurer, d'un côté, les échecs des divers courants féministes à faire des hommes le sujet de leurs luttes politiques, de l'autre, une prise de responsabilité active par les personnages masculins, ce qui ne dénote pas forcément des structures plus démocratiques, mais qui se traduit en tout cas par une plus grande capacité d'adaptation de ces personnages.

\section{Les stratégies de déresponsabilisation des personnages masculins}

5 Durant près d'un demi-siècle, de 1950 à 1990, de nombreuses stratégies narratives déresponsabilisaient les personnages masculins: dans I Love Lucy (CBS, 1951-1957), l'héroïne femme au foyer mais aspirante comédienne se voit opposer des réponses arbitraires à ses aspirations professionnelles; dans The Donna Reed Show (ABC, 1958-1966), la différence sexuelle est régulièrement invoquée pour légitimer la division du travail ; plus généralement, dans Ma Sorcière bien-aimée (ABC, 1964-1972), devant les absences et les fuites de Jean-Pierre, première figure masculine frontalement malmenée, Samantha investit la maison comme un lieu d'expression de ses pouvoirs magiques, métaphore de ses capacités d'agir. Confrontés aux désirs d'émancipation féminine qui percent déjà dans les imaginaires médiatiques, les personnages masculins repoussent tout simplement la discussion, obligeant les femmes qui refusent de se soumettre totalement, à trouver d'autres moyens : cachotteries, secrets, manipulations sont autant de manières de remettre en cause l'autorité masculine sans menacer l'ensemble du système patriarcal. Lorsque les hommes sapent ainsi les capacités d'agir féminines, les femmes retournent à leur avantage la confidentialité de leurs talents et la discrétion de leur champ d'action qu'est la sphère privée en agissant sur un registre clandestin, voire stratégique (ce qui sera exacerbé quelques décennies plus tard dans Desperate Housewives).

6 Ce mouvement de déresponsabilisation a continué de se développer à compter des années 1970 avec la prédominance du féminisme libéral qui, prônant la conquête de la sphère publique, délaissait la critique systémique de la sphère privée (Dow, 1996). Dans 
The Mary Tyler Moore Show (CBS, 1970-1977) comme dans Murphy Brown (CBS, 1988-1998), les personnages masculins sont relégués au deuxième plan, afin de produire des héroïnes au sens fort du terme, c'est-à-dire dont les aventures ne dépendent pas des hommes. Dès les premiers épisodes, Mary Richards a cette formule qui semble être sa critique féministe autant qu'une revendication métatextuelle des créateurs: «je pourrais découvrir le secret de l'immortalité et les gens diraient toujours : 'regardez cette fille seule qui a découvert le secret de l'immortalité !'" (The Mary Tyler Moore Show, S01XE05). Dans la même veine, Murphy Brown choisit d'élever son fils seule, faute de candidats qu'elle juge sérieux. Ces indépendances ont pour paradoxe typique du féminisme libéral de faire reposer l'émancipation féminine sur les seules épaules des femmes, et des femmes issues de classes supérieures de surcroît: célibat et épanouissement professionnel sont un luxe que peuvent se permettre des individus dont le métier n'est pas seulement alimentaire. En parallèle, des séries comme Maude (CBS, 1972-1978) ou plus encore Roseanne (ABC, 1988-1997) représentent des foyers familiaux travaillés de l'intérieur par le féminisme radical, qui agit pour repenser l'ensemble de l'organisation patriarcale. Walter et Dan sont confrontés à des épouses braillardes et revendicatrices et ils expriment régulièrement la peur des conséquences du féminisme. Les risques d'une domination inversée planent sur ces couples, dans lesquels l'émancipation féminine menace sans cesse de se transformer en affirmation vengeresse d'un pouvoir féminin débridé et castrateur.

7 Durant ces quelques décennies, l'ennemi est cerné : ce sont bien les masculinités patriarcales qui sont directement accusées d'enrayer l'émancipation des femmes, sans que celles-ci ne puissent déployer d'autres défenses que des compétences émotionnelles. Il semble si peu possible pour les imaginaires médiatiques de redéfinir ces masculinités que les solutions sont soit d'invisibiliser ces personnages des représentations, ce qui a le mérite de produire des héroïnes indépendantes, soit d'insérer les hommes dans une maison quasi matriarcale où, argument anti-égalitaire des plus classiques, la demande de redistribution du pouvoir menace de se transformer en retournement de la domination.

\section{Des masculinités fracturées}

8 Les premières traces de l'implication des personnages masculins peuvent être trouvées au milieu des années 1990, lorsque des séries comme Ally McBeal (FOX, 1997-2002) et Sex and the City (HBO, 1998-2004) problématisent les valeurs de l'égalité de genre, de la libération sexuelle et de l'individualisme réflexif. Toutefois, cette thématique affleure d'abord, non par la restructuration des sphères publique/privée ni par le rééquilibrage des charges mentales que demandaient par exemple Maude et Roseanne, mais par une dénaturalisation du genre : confrontés à des héroïnes individuellement autonomes et sexuellement libérées, les personnages masculins entrent dans une crise dont les symptômes sont hétérogènes.

9 Certains personnages sont impotents, comme le vampire Angel (Buffy, The WB-UPN, 1997-2001) qui ne peut faire l'amour sous peine de perdre son âme ; comme Leo, l'ange déchu (Charmed, The WB, 1998-2006) ou comme le paraplégique Logan (Dark Angel, FOX, 2000-2002), condamné à des prothèses cyborg comme le fauteuil roulant ou l'exosquelette; d'autres se transforment en misogynes caricaturaux dont les performances de genre sont des mascarades (Billy, Ally McBeal) et, de façon générale, les 
corps masculins subissent une objectivation jusqu'ici réservée aux femmes (Morin, 2014). Les masculinités se fracturent en elles-mêmes à mesure qu'elles entrent en crise, mais aussi entre elles : les performances de genres se multiplient, qui vont désormais de la douceur à la violence machiste - le paroxysme de cette dernière se trouvant probablement dans Buffy où le vampire Spike, dévasté d'avoir été quitté par l'héroïne, tente de la violer (Buffy, S06XE19). Cette double fracture tient à ce que les personnages masculins sont déboussolés par les nouveaux codes genrés de l'après-féminisme et par la redistribution du pouvoir que celui-ci a engendrée.

10 Toutefois, ce trouble dans le genre, selon la formule désormais consacrée, est immédiatement contrecarré par des comportements amoureux qui restent traditionnels. Si les masculinités entrent en crise, ce qui produit des performances de genre qui ne sont plus simplement patriarcales, les personnages ne s'impliquent pas pour autant dans l'égalité de genre. Les hommes représentés dans notre corpus sont peu engagés dans les processus conjugaux, n'évoquent presque jamais de revendications féministes et occupent même des fonctions relatives à la première modernité : ils sont des figures tutélaires, qui arguent bien souvent savoir mieux que les femmes ce qui est bon pour elles (Ally McBeal, Buffy, Sex and the City), et il n'est pas rare qu'ils se découvrent père d'une fillette, ce qui entre alors en concurrence directe avec leur relation amoureuse comme dans Ally McBeal ou Gilmore Girls (The WB-The CW, 2000-2007). Ces situations et ces statuts, dont on peut se demander s'ils sont un dernier sursaut de l'idéologie patriarcale, empêchent le développement d'une communication démocratique. Mr Big refuse d'exprimer ses sentiments à Carrie (Sex and the City); Larry quitte Ally en partant de Boston sans même la prévenir (Ally McBeal); Angel rompt unilatéralement, persuadé qu'un jour et malgré ses dires, Buffy voudra une vie « normale» (Buffy); Luke, grand misanthrope, met presque dix ans à avouer son amour (Gilmore Girls).

11 Ces déséquilibres entre hommes et femmes proviennent majoritairement de l'inégale répartition des charges mentales : du fait de l'assignation historique des femmes à la sphère privée et de leur arrivée tardive dans la sphère publique, les personnages féminins sont majoritairement ceux qui portent le poids de la construction et de la réaffirmation des relations. Cela conduit à une fatigue et une vulnérabilité du féminin, et à une hégémonie émotionnelle du masculin. La participation féminine à la démocratie relationnelle est alors structurée par des compétences émotionnelles fortes mais par une confiance en soi enrayée. Or, le cercle est vicieux : moins les hommes travaillent au maintien de la relation amoureuse, moins les femmes sont sûres d'elles. Le contrecoup est un surinvestissement du champ amoureux. Dans Sex and the City, Mr Big a beau dire clairement quelles sont ses attentes, Carrie force des discussions qui sabotent la relation et qui portent invariablement sur la consolidation de la relation: publicisation de leur relation, exclusivité affective et sexuelle, degré d'attachement... autant de composantes qu'elle prend à sa seule charge, faute de pouvoir en discuter avec son partenaire. Les personnages masculins restent d'autant plus fermés que les femmes les intègrent en fait peu à la construction d'un amour féministe : dans Sex and the City, les discussions sur leur couple ne se font pas tant avec leur partenaire qu'avec les amies. En plus des charges mentales, les héroïnes portent également seules la responsabilité des échecs: s'inquiétant que $\mathrm{Mr}$ Big ne semble pas engagé dans la relation après qu'elle a fait l'amour avec lui dès le premier soir, Carrie culpabilise : «la vérité c'est que je m'en voulais, c'est moi qui ai choisi de porter cette robe dès le premier rendez-vous, c'est moi qui ai couché avec lui trop rapidement» (Sex and the 
City, S01XE06). La sexualité féminine que le néoféminisme revalorise comme un des atouts majeurs des femmes n'est pas aussi libératrice que promise.

Dans les années 1990, les hérö̈nes gardent toujours l'espoir d'être sauvées des affres angoissantes de la réflexivité par un chevalier blanc, et ce parce que l'expression masculine du romantisme est objet du féminisme mais n'en est jamais le sujet. Aussi, ce qui pose problème dans le cas de ces personnages majoritairement trentenaires n'est peut-être pas tant la sur-responsabilisation individualiste du féminin que la sousresponsabilisation féministe du masculin : si les femmes sont presque uniques garantes des relations amoureuses, la raison en incombe non pas à l'individualisme - qui tend plutôt à leur redonner ce dont elles ont été privées (la possibilité de s'épanouir par l'attention à soi et le temps personnel)-, comme le défend notamment Angela McRobbie (2009), mais à l'assignation du féminisme aux femmes.

13 Ce n'est donc pas un hasard si ces héroïnes sont ultra-féminines et consuméristes en même temps qu'elles débordent du cadre traditionnel pour expérimenter des pratiques codées masculines (au premier rang desquelles une sexualité décomplexée), et si les hommes subissent, du fait de la masculinisation grandissante du regard féminin, une forme d'objectivation de leur corps tout en gardant des comportements amoureux distants et hautains. La démocratisation des comportements masculins que récupèrent les femmes apporte certes une dénaturalisation du genre mais elle ne le déconstruit pas ni ne remet profondément en cause le système romantique. En retour, la démocratisation des politiques féministes pour les hommes, et l'intégration de leur responsabilité dans la prévalence du romantisme, apparait comme une étape urgente pour amorcer la démocratie relationnelle.

14 Ce constat d'une sur-responsabilisation des femmes, Bonnie Dow le formule déjà dans Prime Time Feminism (1992). Elle l'impute à la teneur individualiste du postféminisme, qui dissoudrait les politiques féministes dans des choix personnels. En rabattant les problèmes d'égalité de genre sur les identités féminines, l'individualisme trahirait la dimension collective du féminisme de la deuxième vague. En conséquence, la politique traditionnelle serait affaiblie au profit d'une valorisation des choix personnels, sans grande conséquence sur les structures patriarcales. On peut objecter à cette vision celle de François de Singly pour qui « l'individualisme est un humanisme » (2007) : puisqu'il refuse toute assignation identitaire au nom de l'émancipation du sujet, l'individualisme a en fait été un allié historique du féminisme. Il rend les amours certes plus précaires mais aussi plus malléables selon les envies de chacun et surtout de chacune. Ainsi, si la politique est moins militante, elle n'est pas nécessairement moins subversive. Les tournants culturel et réflexif apparaissent main dans la main dans les imaginaires médiaculturels qui, délogeant les héroïnes de l'activisme et les faisant embrasser l'interrogation des liens sociaux, font naître une individualisation de l'action guidée par des micropolitiques.

15 Si les années 1990 signent l'apparition très explicite du mouvement féministe dans les représentations médiatiques, cela ne se fait donc qu'en l'assignant au féminin et en l'interdisant au masculin, hors du cadre militant ou activiste. Or, la question de l'appropriation du féminisme par les hommes devient cruciale. Certes, elle risque de plier en sens inverse le mouvement émancipateur mais, en retour, laisser le masculin au stade de l'objet de discussion du féminisme revient à laisser ce dernier à la seule charge des femmes et à entériner la déresponsabilisation des hommes, sans parler du fait que, comme le montre Patrick Hopkins (2008), l'idée que le féminisme serait 
antinomique du masculin cache un essentialisme latent. L'opposition putative entre hommes et féminisme repose en effet sur l'opposition binaire entre le genre masculin (qui serait consubstantiel à la domination masculine) et le genre féminin (Digby, 2008).

\section{Les récentes apories des masculinités}

16 Il faut attendre les années 2000 pour que les hommes deviennent explicitement responsables des inégalités de genre. Dans des séries qui représentent bien souvent un monde post-romantique, les personnages masculins deviennent non seulement faillibles, mais de surcroît leurs tromperies, mensonges et insouciances domestiques ne sont plus protégés par la dichotomie classique entre public et privé, ni tolérés par les héroïnes.

Des maris jusqu'aux pères, les hommes sont soit absents soit irresponsables, et le masculin s'incarne en un nouveau personnage : le frère de l'héroïne. Owen surgit aux moments sensibles pour dire à sa sœur ce qu'elle refuse d'admettre (The Good Wife) ; Sean est une béquille certes tremblante pour l'héroïne (The Big $C$, Showtime, 2010-2013) ; la relation quasi incestueuse qu'entretient Christine avec Matthew est un ressort comique, ce dernier occupant d'autant plus la place du petit-ami qu'il habite dans une dépendance de la maison (The New Adventures of Old Christine, CBS, 2006-2010). Les masculinités et les orientations sexuelles de ces frères agissent à contrepied des exépoux souvent virils ou masculinistes: Owen est homosexuel et défend des politiques progressistes; Sean, bipolaire, est un activiste environnemental ; Matthew entretient avec sa mère une relation abusive (l'appelant tous les jours et l'embrassant sur la bouche) et ne sait quoi faire de sa vie professionnelle. La présence insistante de ces personnages aux masculinités troublées comble souvent la place laissée vacante par des amitiés devenues éparpillées.

Dans le domaine amoureux, pour la première fois, les personnages masculins doivent s'adapter aux mutations féministes des héroïnes. Quand le care féminin est concurrencé par l'émancipation, le partage des tâches domestiques, des charges mentales et des marques affectives devient impératif. Autonomie et responsabilité sont redistribuées aux hommes en même temps que liberté et épanouissement deviennent pour les femmes, non pas une priorité absolue, mais une variable qui n'est plus négociable. D'un côté, les personnages masculins participent de plus en plus à l'élaboration du couple, non sans difficultés, comme dans The Good Wife (CBS, 2009-2015) où Peter peine à se détacher de ses habitudes patriarcales (ne comprenant pas, par exemple, pourquoi Alicia met tant de temps à pardonner ses infidélités) et où Will est surpris qu'Alicia reste insensible à ses envolées lyriques. D'un autre côté, corollaire de la responsabilisation masculine, les femmes découvrent les vertus de l'égoïsme. Dans Cougar Town (ABC-TBS, 2009-2015), l'égocentrisme est favorablement redéfini en ce qu'il décentre l'obsession de la perfection pour privilégier l'authenticité. Le sacrifice des envies et des plaisirs, qui était tant exhorté dans les sitcoms familiales des années 1950 et 1960 , devient contre-productif à l'heure où, selon les valeurs individualistes, le respect des autres passe par le respect de sa propre personne.

Les réponses se scindent en deux principaux camps qu'incarnent des personnages aux performances de genre toutefois très hétérogènes: d'un côté, la «crise de la masculinité » de la décennie précédente se poursuit, à cela près que pour y répondre, les hommes déploient désormais des formes de manipulations stratégiques ou 
émotionnelles (Desperate Housewives, ABC, 2004-2014; Ugly Betty, ABC, 2006-2010; Damages, FX, 2007-2010; The Good Wife, CBS, 2009-2015) historiquement réservées aux personnages féminins : pour obtenir un enfant de son épouse, Carlos décide de truquer sa plaquette de pilules (Desperate Housewives). En parallèle à ces figures patriarcales que les femmes accusent directement d'être à l'origine de leur oppression, émergent de nouveaux personnages masculins qui, au contraire, assument d'eux-mêmes leur responsabilité dans l'égalité de genre. Au-delà de cette opposition somme toute classique entre résistances et collaborations, dans un cas comme dans l'autre, les redéfinitions, qu'elles soient de l'ordre des codes communicationnels, des performances de genre ou des attentes amoureuses et sexuelles, opèrent sur le mode du féminin.

Dans ce deuxième ensemble, les personnages masculins performent des subjectivités plus sensibles et se révèlent attachés à la construction de démocraties relationnelles empreintes de négociations et de compromis (The Good Wife, CBS, 2009-2015; Nurse Jackie, Showtime, 2009-2015 ; Parks and Recreation, NBC, 2009-2015 ; Jane the Virgin, The CW, 2014-). Les codes discursifs réservés à ces nouvelles masculinités leur confèrent une forte valorisation et la contradiction semble dépassée entre masculinité et émotion. Des personnages plus vulnérables apparaissent, à qui l'on consacre des scènes introspectives et des séquences poétiques: ils se plaignent d'être esseulés (The Good Wife), d'être pris entre le devoir de poursuivre l'entreprise paternelle et la volonté de s'épanouir émotionnellement (Jane the Virgin) ou encore de ne pas être suffisamment impliqués dans les décisions familiales (Nurse Jackie). Dans Cougar Town, une issue à la domination féminine que subit Andy et à l'échec social de Bobby, gentil loser, est trouvée dans l'homosocialité des deux personnages.

21 Le manque d'amour de ces hommes est désormais clairement exprimé. Il peut être tour à tour une punition de la réussite professionnelle (ce qui était là aussi une caractéristique jusqu'ici réservée aux héroïnes: Murphy Brown, Sex and the City, Ally McBeal, Buffy, Charmed, Alias, Dark Angel...), comme pour Will (The Good Wife) dont l'ambition capitaliste est compensée par une fragilité émotionnelle ; il peut également être une fragilité due à une figure paternelle écrasante (Jane The Virgin); ou enfin, être directement dû à la redistribution des compétences comme dans Nurse Jackie où l'agir communicationnel de Kevin, tourné vers le compromis, heurte de plein fouet l'agir stratégique de Jackie, capable de manipuler son époux pour parvenir à ses fins. Les insatisfactions exprimées dans le couple sont typiquement féminines : sentiment d'être délaissé, colère de n'être jamais consulté, reproches quant à l'absence et au manque d'implication de son épouse dans la vie de famille. Il est intéressant de voir que dans les simples disputes comme dans les cas les plus radicaux d'inversion de l'hégémonie (Nurse Jackie), à aucun moment les personnages masculins n'incriminent le féminisme ni même l'épanouissement individuel des femmes. Ces reproches sont réservés aux enfants, signe que le care maternel reste inquestionné là où le care conjugal est mis à mal (The Good Wife, Nurse Jackie, Desperate Housewives).

Le sentiment d'être délaissé est prégnant, ce qui pourrait être symboliquement compris comme, d'une part, la mise à l'écart de l'agenda féministe, et d'autre part, la difficulté à se convertir au dialogue démocratique auquel les enjoignent les femmes : les héroïnes, accablées par les difficultés de l'émancipation, souvent confrontées par ailleurs à des personnages toujours patriarcaux, croulant toujours sous les responsabilités culpabilisantes de la maternité, ont peu de temps à consacrer à ces personnages 
sensibles. Lorsqu'elles le font, c'est exclusivement sur un mode introspectif encore difficile pour des personnages masculins dont les performances de genre restent souvent viriles. Si les émotions masculines sont ressenties et montrées, elles sont donc peu assumées et peu communiquées. C'est un monde de psychologie généralisée qui est ainsi représenté, s'étendant des personnages féminins aux masculins, dans lequel, pour la première fois, les femmes opèrent des échanges entre compétences professionnelles et compétences amoureuses, tandis que les hommes rencontrent de nouveaux défis comme la démocratie relationnelle et l'expression des ressentis, et dont la particularité est qu'ils restent, pour l'heure, sans réponse. On voit bien à cet égard la place cruciale qu'occupe la communication dans des relations qui ne sont plus seulement légitimées par la structure maritale... mais les hommes surnagent, sans trouver de réinventions satisfaisantes.

\section{Conclusion}

Dans les séries étatsuniennes, l'idée d'hommes féministes est longtemps restée à l'état de pur impensé soutenu par une division fonctionnaliste de la société : les quelques contaminations qui ont lieu, comme lorsque l'époux de Lucy (I Love Lucy) expérimente le labeur domestique, défendent in fine un "juste retour des choses ». Puis, lorsqu'à l'orée des années 1970, l'émancipation féminine pénètre tant dans les imaginaires médiatiques que les masculinités ne peuvent plus l'ignorer, l'oxymore est tel que les séries préfèrent rayer ou invisibiliser les hommes de leurs récits. Après cette période où les représentations médiatiques montrent des femmes ignorées ou stratégiquement esseulées, symptômes d'une incapacité à faire percer des modèles plus démocratiques, les séries télévisées s'emparent de façon plus hétérogène du problème. L'innovation repose sur l'apparition de personnages masculins qui expérimentent une nouvelle sensibilité, sans avoir véritablement les outils ou la place pour la communiquer.

Voit-on ici poindre des masculinités post-patriarcales? Insistant sur le potentiel subversif du féminisme radical, qui enjoint aux hommes de refuser l'assignation de genre ainsi que la hiérarchisation et la visée hégémonique qui en résulte, Sandra Harding explique qu'ils " peuvent refuser de respecter les idéaux de la masculinité qui structurent les cultures, les politiques et les pratiques de tant d'institutions sociales » (2008: 181). Cela implique en retour d'interroger les statuts de sujet et d'objet du féminisme : faire des femmes l'unique sujet du féminisme aura été une étape cruciale de la construction du sexisme comme problème public, mais elle est une étape qu'il semble être temps de dépasser au risque de retomber dans de nouvelles formes d'essentialisme. L'une des plus dangereuses est celle du procès d'intention, qui consiste, de façon déterministe et réductionniste, à présupposer que la position sociale masculine induit mécaniquement telle ou telle position politique. Un tel projet a pour écueil d'exalter en retour les qualités des femmes du simple fait qu'elles sont dominées, ce que cristallise le courant néoféministe... qui n’a plus de féministe que le nom. En retour, ouvrir le féminisme aux hommes ne relève pas d'un humanisme angéliste qui ignorerait les intérêts contradictoires des groupes sociaux, mais d'une stratégie politique de déconstruction des genres et de redistribution des pouvoirs. 


\section{BIBLIOGRAPHIE}

BECK Ulrich et Elisabeth BECK-GERNSHEIM (1995), The normal chaos of love, Cambridge, Polity.

DIGBY Tom (2008), Bartky, Sandra, Men Doing Feminism, Londres, Routledge.

Dow Bonnie J. (1996), Prime-Time Feminism. Television, Media Culture, and the Women's Movement Since 1970, Philadelphia, University of Pennsylvania Press.

GIDDENS Anthony (1992 [2004]), La transformation de l'intimité. Sexualité, amour et érotisme dans les sociétés modernes, traduit de l'anglais par Jean MOUCHARD, Rodez, Le Rouergue/Chambon.

HOPKINS Patrick (2008), « How Feminism Made a Man Out of Me: The Proper Subject of Feminism and the Problem of Men ", dans Tom DIGBY, et Sandra BARTKY, Men Doing Feminism, Londres, Routledge.

HARDING Sandra (2008), « Can Men Be Subject of Feminism? », dans Tom DIGBY, et Sandra BARTKY, Men Doing Feminism, Londres, Routledge.

MCROBBIE Angela (2009), The Aftermath of Feminism. Gender, Culture and Social Change, Londres, Sage Publications.

MORIN Céline (2014), « Sexualités et redistribution des pouvoirs dans les séries américaines », Hermès, $n^{\circ} 69$, p. 97-101.

SINGLY de François (2007), L'Individualisme est un humanisme, Paris, Éditions de l'Aube.

\section{RÉSUMÉS}

Cet article analyse la lente responsabilisation des personnages masculins de séries étatsuniennes quant aux inégalités de genre. Après un court retour sur quarante ans d'imaginaires médiatiques, sont explorées les premières traces de l'implication masculine au milieu des années 1990 : confrontés à des héroïnes individuellement autonomes et sexuellement libérées, les personnages masculins entrent dans une crise dont les symptômes sont hétérogènes, bien que les comportements amoureux restent traditionnellement répartis. À compter des années 2000 , en parallèle de figures patriarcales que les femmes accusent d'être à l'origine de leur oppression, émergent de nouveaux personnages masculins qui assument d'eux-mêmes leur responsabilité dans l'égalité de genre. Ces hommes, aux subjectivités plus sensibles, sont confrontés à de nouveaux défis qui restent pour l'heure insolubles.

This article focuses on the slow process of involvement of male characters in American television series regarding gender inequalities. After a brief historical review, the article analyzes the first participations made by male characters in the mid-90s. When confronted with individualist and sexually liberated heroines, male characters enter a crisis whose symptoms are heterogeneous while love behaviours remain traditionally distributed. Beginning in the 2000s, in parallel with patriarchal figures that women directly accuse of being the source of their oppression, new male characters appear who the particularity of assuming responsibility in the struggle for gender equality. These representations show men with more sensitive subjectivities facing new and various challenges which remain, for now, unsolvable. 
INDEX

Keywords : masculinities, feminist movements, love, public sphere, representations

Mots-clés : masculinités, féminismes, amour, sphère publique, représentations

\section{AUTEUR}

\section{CÉLINE MORIN}

Céline Morin est docteure en Sciences de l'information et de la communication, rattachée au laboratoire CIM/MCPN. Sa thèse, qui porte sur les représentations des amours des héroïnes de séries télévisées américaines de 1950 à 2010, analyse la montée de problématiques égalitaires, que matérialise le modèle de la « relation pure ». Elle a notamment publié dans Le Temps des médias, Hermès et Questions de communication. 